\title{
Altered Intestinal Microbiomes and Lipid Metabolism in Patients With Chronic Disorders of Consciousness
}

Benyan Luo ( $\sim$ luobenyan@zju.edu.cn )

Zhejiang University School of Medicine First Affiliated Hospital https://orcid.org/0000-0002-9892-5778

Jie Yu

Zhejiang University School of Medicine First Affiliated Hospital

Qisheng Cheng

Zhejiang University School of Medicine First Affiliated Hospital

Fangping He

Zhejiang University School of Medicine First Affiliated Hospital

\section{Fanxia Meng}

Zhejiang University School of Medicine First Affiliated Hospital

Yamei Yu

Zhejiang University School of Medicine First Affiliated Hospital

Chuan Xu

Zhejiang University School of Medicine First Affiliated Hospital

\section{Xinrui Wen}

Zhejiang University School of Medicine First Affiliated Hospital

\section{Lirong Hong}

Hangzhou hospital of zhejiang armed police corps

Jian Gao

Hangzhou mingzhou brain Rehibitation hospital

Jingqi Li

Hangzhou mingzhou brain Rehibitation hospital

\section{Gang Pan}

State Key Laboratory of CAD and CG

Ming D. Li

Zhejiang University First Affiliated Hospital State Key Laboratory for Diagnosis and Treatment of Infectious Diseases

\section{Research article}

Keywords: Chronic disorders of consciousness, intestinal microbiome, short chain fatty acid, brain functional connectivity, biomarkers

Posted Date: August 24th, 2021

DOI: https://doi.org/10.21203/rs.3.rs-824411/v1

License: @ (1) This work is licensed under a Creative Commons Attribution 4.0 International License. Read Full License 


\section{Abstract}

Intestinal microbiotas regulate brain function of the host through the production of a myriad of metabolites and are associated with various neurological diseases. Understanding intestinal microbiome of patients in chronic disorders of consciousness (DoC) is important for the evaluation and treatment of the disease. To investigate the difference of intestinal microbiome and short-chain fatty acids (SCFAs) among the patients in vegetative state (VS), minimally conscious state (MCS) and emerged from minimally conscious state (EMCS), as well as the influence of antibiotics on these patients, 16S rRNA sequencing and targeted lipidomics were performed on their fecal samples and EEG signals analysis were used to evaluate their brain function. Our results showed that intestinal microbiome among the three groups differed greatly and some microbial community with its production of SCFAs were reduced in VS patients compared to the other two groups. Moreover, reduced microbial communities and five major SCFAs along with attenuated brain functional connectivity were observed in MCS patients treated with antibiotics compared to those received no antibiotic treatment, but not in the other pairwise comparisons. Finally, three genera level of microbiota, Faecailbacterium, Enterococcus and Methanobrevibacter, were considered as potential biomarkers to distinguish patients in MCS from VS with high accuracy both in the discovery cohorts and validation cohorts. Together, our findings improved the understanding of patients with chronic DoC from intestinal microbiome and provided a new reference for therapeutic targets exploration.

\section{Background}

Patients who survived severe traumatic brain injury (TBI) usually enter chronic disorders of consciousness (DoC), which contain two major types: vegetative state (VS) and minimally conscious state (MCS)[1-3]. VS patients retained the behavioral sleep-wake cycle but present with unaware of themselves or their environment, whereas MCS patients have reproducible signs of awareness and exhibit fluctuations in consciousness[4, 5]. In addition, patients who recover functional object uses or communication from VS or MCS are referred to as emerged from minimally conscious state (EMCS)[5]. Different from brain death, these three types of patients retain brain activity[6] and do not dependent on a ventilator for survival. They all received enteral nutrition. Over the last few decades, numerous evaluations and treatments have been performed for the central nervous system of patients with chronic DoC[7]. However, little attention has been paid to its peripheral physiological environment, including intestinal microecology, which can also influence the brain function.

The intestinal microbiota can modify and regulate various chemical signals from the environment by gut-microbiota-brain axis that then circulate throughout the body[8]. Recently it has been demonstrated that intestinal microbiota may directly influence human health and associate with many neurological diseases[9]. In addition, short-chain fatty acids (SCFAs) are lipids produced by some intestinal microbiota through fermentation of dietary fiber that can act on the brain by regulating neuroplasticity and the immune system[10]. For example, propionic acid of serum and feces of with multiple sclerosis patients reduced significantly compared with healthy subjects and reduction of annual relapse rate, disability stabilization, brain atrophy after 3 years of propionic acid intake[11]. Therefore, investigation of the difference of intestinal microbiota and SCFAs amounts in patients with different levels of consciousness can understand its relationship to the restoration of brain function and consciousness, as well as found potential therapeutic targets related to intestinal microbiota.

Recently, intestinal microbiome have been widely applied to explore the difference of intestinal microbiota during the occurrence and development of disease, which substantially contributed to our understanding of patients-microbiota interactions[12]. In our previous studies, high-throughput sequencing of bacterial 16S rRNA was used for investigation of intestinal microbiome in patients with cognitive impairment and found it closely associate with brain functional connectivity of them[13, 14]. However, the relationship between chronic DoC patients and their intestinal microbiota have been still unexplored. To address this knowledge gap, we examined both intestinal microbiome by 16S rRNA sequencing and SCFAs by targeted lipidomics in fecal samples specific to different levels of consciousness. Moreover, intermittent use of antibiotics are usually performed for the TBI patients to prevent a wide variety of infections, especially ventilator-associated pneumonia in clinical[15]. So, we further compared the difference of intestinal microbiome and brain functional connectivity between the chronic DoC patients treated by antibiotic or not, with the ultimate goal of exploring the alteration of antibiotics on intestinal microbiota of the patients with different levels of consciousness and whether this alteration can influence the brain function restoration and prognosis of these patients. Finally, we identified potential biomarkers that can distinguish MCS from VS patients and EMCS from MCS patients both in the discovery cohorts and validation cohorts.

\section{Material And Methods}

2.1 Ethics statement: Written informed consent was obtained from each patient's legal guardian. This study was approved by the Ethics Committee of the First Affiliated Hospital, School of Medicine (No. NCT04530968), Zhejiang University, Hangzhou Hospital of Zhejiang Armed Police Corps, China, and Hangzhou Mingzhou Brain Rehabilitation Hospital.

2.2 Subjects: Patients after severe traumatic brain injury (TBI) were recruited from the rehabilitation units of Hangzhou Hospital of Zhejiang Armed Police Corps, China, and Hangzhou Mingzhou Brain Rehabilitation Hospital, including discovery cohorts: VS ( $n=13$; average age

Page $2 / 20$ 
50.7 \pm 13.2 years; 9 males and 4 females), MCS ( $n=15$; average age 49.0 \pm 9.8 years; 10 males and 5 females) and EMCS ( $n=15$; average age $47.7 \pm 12.1$ years; 12 males and 3 females); validation cohorts: VS ( $n=14$; average age $57.5 \pm 10.5$ years; 10 males and 4 females), MCS ( $n=13$; average age $56.3 \pm 14.2$ years; 10 males and 3 females) and EMCS ( $n=17$; average age $53.0 \pm 16.7$ years; 11 males and 6 females). Glasgow Coma Scale (GCS) was used for assessment of the severity of brain injury in acute stage and Glasgow Outcome Scale-Expended (GOS-E) was used for assessing their prognosis at 6 months[16]. Diagnosis of VS, MCS and EMCS based on the five assessments within 10 days by DoC experts using the Coma Recovery Scale-Revised (CRS-R)[5]. In brief, VS: can open their eyes and preserve sleep-wake cycles, but unaware of themselves and their surroundings; MCS: have reproducible signs of awareness and exhibit fluctuations in consciousness; EMCS: recovery of functional object uses or communication from chronic DoC; all patients maintained this physiological state more than 1 month prior to enrollment and accepted the diet provided in the hospital for a long time; all the patients received enteral nutrition from the hospital.

Patients with other causes of DoC and unstable consciousness state (There are signs of spontaneous recovery or deterioration within 1 week) were excluded from this study. Subjects who had the history of using antibiotics, probiotics, prebiotics, or synbiotics within one month before fecal sample collection; or with severe malnutrition, infection, drug or alcohol addicts; irritable bowel syndrome; inflammatory bowel disease in the last year; and people with schizophrenia, schizoaffective disorder or primary affective disorder; combined severe heart, brain, liver, kidney and hematopoietic system diseases or other serious primary diseases. Patients who received some special treatment, such as transcranial magnetic stimulation (TMS) and transcranial direct current stimulation (tDCS) were also excluded. The demographic and clinical characteristics of the subjects were listed in Table 1 and Table S4.

The other three groups of patients who had the history of using antibiotics but conform to other exclusion criteria were also recruited from these same hospitals: VS with antibiotics (VS-Abx, $n=21$; average age 54.9 \pm 11.8 years; 15 males and 6 females); MCS with antibiotics (MCSAbx, $n=17$; average age $62.8 \pm 8.7$ years; 12 males and 5 females); EMCS with antibiotics (EMCS-Abx, $n=9$; average age $61.0 \pm 11.6$ years; 5 males and 4 females). The demographic and clinical characteristics of the subjects were listed in $\underline{\text { Table } 1 .}$

All subjects were categorized based on their CRS-R scores calculated and centrally acting drugs, neuromuscular function blockers and sedation were discontinued for at least 24 hours when blood samples were drawn[17]. Fasting plasma samples of all patients and were collected in the day of fecal sample collection. Patients intact skulls were also accepted for resting EEG recording.

2.3 Sample Collection and Extraction of genome DNA: Fecal samples from EMCS, MCS and VS patients were collected at admission. Fecal samples were processed in the laboratory within 4 hours after collection All fecal samples within half an hour dispensed in $2 \mathrm{ml}$ Eppendorf tubes, each tube packing $180 \pm 20 \mathrm{mg}$, immediately placed in $-80^{\circ} \mathrm{C}$ until analysis. Total genome DNA from samples was extracted using cetyltrimethylammonium bromide (CTAB) and sodium dodecyl sulphate (SDS) method[18]. DNA concentration and purity were monitored on $1 \%$ agarose gels. According to the concentration, DNA was diluted to $1 \mathrm{ng} / \mu \mathrm{L}$ using sterile water.

2.4 Amplicon Generation, Polymerase chain reaction (PCR) and sequencing: The isolated bacterial genomic DNA was used as a template for PCR amplification of V3-V4 region of the bacterial 16S ribosomal RNA gene in a multiplex approach with the forward primers. All PCR reactions were carried out with $15 \mu \mathrm{L}$ of Phusion ${ }^{\circledR}$ High-Fidelity PCR Master Mix (New England Biolabs); $0.2 \mu \mathrm{M}$ of forward and reverse primers, and about $10 \mathrm{ng}$ template DNA. Thermal cycling consisted of initial denaturation at $98^{\circ} \mathrm{C}$ for 1 min, followed by 30 cycles of denaturation at $98^{\circ} \mathrm{C}$ for $10 \mathrm{~s}$, annealing at $50^{\circ} \mathrm{C}$ for $30 \mathrm{~s}$, and elongation at $72^{\circ} \mathrm{C}$ for $30 \mathrm{~s}$. Finally, $72{ }^{\circ} \mathrm{C}$ for 5 min. Mix same volume of $1 \times$ loading buffer (contained SYB green) with PCR products and operate electrophoresis on $2 \%$ agarose gel for detection. PCR products was mixed in equidensity. Then, mixture PCR products was purified with Qiagen Gel Extraction Kit (Qiagen, Germany). Sequencing libraries were generated usingTruSeq ${ }^{\circledR}$ DNA PCR-Free Sample Preparation Kit (Illumina, USA) following manufacturer's recommendations and index codes were added. The library quality was assessed on the Qubit@ 2.0 Fluorometer (Thermo Scientific) and Agilent Bioanalyzer 2100 system. At last, the library was sequenced on an Illumina NovaSeq platform and 250 bp paired-end reads were generated.

2.5 Short-chain fatty acid measurements: Samples were thawed on ice, and diluted 1:10 in sterile phosphate-buffered saline solution (PBS) $\$ vortexed for $1 \mathrm{~min}$, and centrifuged at $1000 \mathrm{x}$ g for $2 \mathrm{~min}$ at $4^{\circ} \mathrm{C}$. As our previous study described[19, 20], supernatant was recovered, and pellet was extracted with isopropyl ether. $100 \mu \mathrm{L}$ aliquots were added into a 2 -mL glass centrifuge tube mixing with $50 \mu \mathrm{L}$ of water with $15 \%$ phosphoric acid and $150 \mu \mathrm{L}$ of $5 \mu \mathrm{g} / \mathrm{mL}$ 4-methyl valeric acid (IS). The suspensions were homogenized with a vortex for about 1 min and centrifuged for $10 \mathrm{~min}$ at $12000 \times \mathrm{g} .1 \mu \mathrm{l}$ supernatant was taken for GC-MS analysis using an Agilent Model 7890A/5975C GC-MS system. To quantify Short-chain fatty acid, a calibration curve for the concentration range of $0.1-100 \mathrm{ug} / \mathrm{ml}$ was constructed. The IS was used to correct for injection variability between samples and minor changes in the instrument response.

The samples were separated with an Agilent HP-INNOWAX capillary GC column $(30 \mathrm{~m} \times 0.25 \mathrm{~mm}$ ID $\times 0.25 \mu \mathrm{m})$. The initial temperature was $90{ }^{\circ} \mathrm{C}$ and was increased to $120^{\circ} \mathrm{C}$ at $10{ }^{\circ} \mathrm{C} / \mathrm{min}$, after which the temperature was increased to $150{ }^{\circ} \mathrm{C}$ at $5{ }^{\circ} \mathrm{C} / \mathrm{min}$ and then to $250{ }^{\circ} \mathrm{C}$ at $25^{\circ} \mathrm{C}$ $/ \mathrm{min}$, where it remained for $2 \mathrm{~min}$. The carrier gas was helium $(1.0 \mathrm{~mL} / \mathrm{min})$. The temperatures of the injection port and transmission line were 
$250{ }^{\circ} \mathrm{C}$ and $230^{\circ} \mathrm{C}$ respectively. The electron bombardment ionization source, SIM $\bowtie$ Selected ion Monitor冈scanning mode, and electron energy were $70 \mathrm{eV}$.

2.6 Data processing: Sequences analysis were performed by Uparse software[21]. All reads were deposited and grouped into operational taxonomic units (OTU) at a sequence identity of $97 \%$, and the taxonomic affiliation of the OTUs was determined with quantitative insights into microbial ecology (QIIME, version 1.8.0) against the Greengenes database version 13.8[22]. The following downstream data analyses were conducted in R software. Alpha diversity is applied in analyzing complexity of species diversity for a sample through 4 indices, including Chao1, Shannon, Simpson, ACE. All these indices in our samples were calculated with QIIME (Version 1.7.0) and displayed with R software (Version 2.15.3). Principal Coordinate Analysis (PCoA) used unweighted, weighted UniFrac distance matrix and Bray-Curtis distance matrices[23]. The linear discriminant analysis (LDA) effect size (LEfSe) method was used to characterize the taxa with statistical significance and biological relevance. For LEfSe analysis, the Kruskal-Wallis test (alpha value of 0.05) and LDA score of $>4$ were used as thresholds. Pairwise comparisons were analyzed with Mann-Whitney U test. Partial least-squares-latent structure discriminate analysis (PLS-DA) was performed by using MetaboAnalyst 4.0 (www.metaboanalyst.ca) to observe the fecal microbiota structure in different groups[13] based on OTUs of the sequencing data from each sample. Variable importance in projection (VIP) reflects the importance of the variables that have the most significant contribution in discriminating. Variables with VIP $>1$ are important contributors to generation of the model. Based on Kyoto Encyclopedia of Genes and Genomes (KEGG) functional pathway, Phylogenetic Investigation of Communities by Reconstruction of Unobserved States (PICRUSt)[24] were used to predict functional composition of the intestinal microbiome for each sample.

2.7 EEG Data Recordings and Analysis: EEG signals were recorded using a 64-electrodes BrainCap (Brain Products DmbH, Munich, Germany) in the international 10-20 system, and 1 of the 64 electrodes was placed under the right eye to record electrooculogram (EOG). Electrode impedances were kept below $10 \mathrm{k} \Omega$. The signals were sampled at $1 \mathrm{kHz}$. EEG signals were referenced online to FCz, but were referenced offline to a common average reference[25]. Raw EEG data were digitally filtered between 0.1 and $46 \mathrm{~Hz}$ (band-pass filter). A baseline correction was also applied to all channels. EEG epochs with ocular, muscular, and other artifacts were visually identified and manually rejected[26]. This was followed by an independent component analysis (ICA; Infomax algorithm) to remove periodically recurring artifacts including horizontal eye movements, blinks, and ECG artifacts. All preprocessing and analysis in this study were conducted in the MATLAB software (The MathWorks, Natick, MA).

A frequency spectrum was. EEG power spectrum density (PSD) were generated using the Welch's method (one of the Fourier transforms)[26]. The different frequency bands on the frequency spectrum were divided by $0.1-46 \mathrm{~Hz}$ to obtain the relative powers. Power values were then averaged across each frequency band: delta $(1-4 \mathrm{~Hz})$, theta $(4-8 \mathrm{~Hz})$, alpha $(8-12 \mathrm{~Hz})$ beta $(12-30 \mathrm{~Hz})$ and gamma $(31-45 \mathrm{~Hz})$ and were averaged across all trials.

Functional connectivity assesses functional communication between brain regions by estimating the level of synchronization of the EEG signals[27]. The phase lag index (PLI) aimed to obtain a measure that provides reliable estimates of phase synchronization between two signals and is insensitive to volume conduction[28]. Here, the instantaneous phases were obtained by initially bandpass filtering the signals within the frequency bands defined above and subsequently using the Hilbert transform to obtain the phase of the corresponding analytic signal[28, 29]. Phase difference distribution $(1 \varphi)$ as an index of asymmetry between a given pair of channels, that were wrapped in the interval, can be obtained in the follow way:

$$
P L I_{x y}=\mid<\operatorname{sgn}(\Delta \varphi(\tau))>1
$$

Different from PLI, the weighted phase lag index (WPLI) improved measure of phase synchronization for electrophysiological signals in the presence of noise and volume conduction and it weighted the contribution of the observed phase leads and lags by the magnitude of the imaginary component of the cross-spectrum[28].

$$
W P L I=\frac{|<\operatorname{Imag}(\operatorname{Sxy}(f))>|}{<|\operatorname{Imag}(\operatorname{Sxy}(f))|>}=\frac{|<| \operatorname{Imag}(\operatorname{Sxy}(f)) \mid \cdot \operatorname{sgn}(\operatorname{Imag}(\operatorname{Sxy}(f))>\mid}{<|\operatorname{Imag}(\operatorname{Sxy}(f))|>}
$$

PLI and WPLI range between 0 and 1, with 0 indicating no correlation and 1 maximal correlation. The connectivity matrices were entered as repeated-measures dependent variables into the network-based statistic (NBS) toolbox[30]. All of the analysis were conducted in the MATLAB software (The MathWorks, Natick, MA).

2.8 Statistical analysis: Continuous variables were reported as means \pm standard error of mean, and statistical comparisons were made using one-way analysis of variance (ANOVA) followed by post hoc Least-Significant Difference (LSD) or the independent t-test. Non-normally distributed variables were expressed as interquartile range (IQR), and comparisons were conducted using the Mann-Whitney U test, KruskalWallis Test or Chi-square tests. Receiver-operating characteristic (ROC) analyses and least absolute shrinkage and selection operator (LASSO)

Page $4 / 20$ 
regression were constructed to calculate the best cutoff point and area under the curve (AUC) for the candidate biomarkers. For correlation analysis, Spearman's rank test was performed. Statistical analysis was performed using SPSS version 23.0. $P$ values of less than 0.05 after multiple-comparison correction using the false discovery rate method were considered significant.

\section{Results}

\section{Clinical characteristics of the study cohorts}

In this study, 43 participants with non-antibiotics interventions and 47 participants with antibiotics interventions, including EMCS patients MCS patients and VS patients, were recruited for 16S rRNA sequences of fecal samples. Demographic and clinical characteristics of the study cohorts are summarized in Table 1. As shown, there was no significant difference among the three groups with respect to age, sex and length of hospital stay. We also recorded and compared the main biochemical indicators of patients on the day fecal samples were collected. There was also no significant difference in leucocyte, neutrophil, lymphocyte, monocyte, platelet, C-reactive protein, glutamic-pyruvic transaminase (GTP), glutamic-pyruvic transaminase (GOT), lactate dehydrogenase (LDH), homocysteine among the three groups with or without antibiotics interventions.

However, VS patients showed significantly lower level of hemoglobin compared to MCS patients and EMCS patients, whether treated with antibiotics or not. Levels of creatinine were lower only in VS patients with non-antibiotics compared the other two groups. In order to exclude the influence of the severity of brain injury in acute stage, we compared the GCS of the patients when they were admitted in the ICU and found no significant difference among three groups with or without antibiotics.

\section{Alterations of microbiomes in VS, MCS and EMCS patients with non-antibiotics treatment}

To assess the alteration of intestinal microbiome in EMCS, MCS and VS patients, all samples were performed by 16S rDNA gene sequencing. As shown in the Venn diagram, 971 OTUs were identified and 428 OTUs of these were shared among all samples. Individuals of MCS and VS shared 515 of 919 OTUs, EMCS and VS shared 497 of 766 OTUs, EMCS and MCS shared 464 of 877 OTUs, respectively (Fig. 1A). Although there was no significant difference in microbial diversity (Shannon and Simpson diversity index) among the three groups (Fig. 1F-G), the mean community richness (ACE and Cha01) were higher in VS and MCS groups compared to EMCS group (Fig. 1D-E). Further we screened top 10 OUTs with the highest relative abundance by ternary plot. The relative abundance of Streptococcus, Lactobacillus and Megamonas was significantly higher in EMCS, MCS and VS patients, respectively (Fig. 1H), suggesting that the interior structure of intestinal microbiome were also altered in the patients with different levels of consciousness. Then we made feature selection to identify gut microbiota that maximized separation among the EMCS, MCS, and VS groups via partial least squares discriminant analysis (PLS-DA) (Fig. 1B). We also performed principal coordinate analysis (PCoA) and cluster analysis (Supplementary Fig. 1A and Fig. 1C), which is consistent with the results of our PLSDA: the samples from the MCS group were located between the EMCS and VS groups but were closer to those of the EMCS group (Supplementary Fig. 1A). Furthermore, we identified the importance of fecal microbiota in PLS-DA model based on Variable Importance in Projection (VIP) score, which is the weighted sum of squares of the PLS loadings for that variable[19]. 38 OUTs were identified based on a VIP score > 2 (Supplementary Fig. 1B). Of these fecal microbiota, Lactococcus, Megamonas, Enterococcus etc enriched in VS group Allisonella, Mogibacterium, Campylobacter etc enriched in EMCS group, while most intestinal microbiota showed lower levels in MCS group compared to those in the other two groups (Supplementary Fig. 1C).

\section{Fecal microbiota taxonomic differences among the EMCS, MCS and VS patients}

To investigate specific alteration of the fecal microbiota of patients with different levels of consciousness, we assessed relative abundance in EMCS, MCS and VS groups at the phylum, class, family, and genus levels (Fig. 2 and Fig. 3A). In our study, none of fecal microbiota at the phylum level showed significant difference among the three groups. At class level, VS patients showed a marked decrease in the relative abundance of Clostridia compared to MCS and EMCS groups, while the relative abundance of Fusobacteriia showed alteration only between MCS group and EMCS group. Conversely, Deltaproteobacteria, the intestinal microbiota associated with inflammatory response[31], performed higher relative abundance in VS group than the other two groups (Fig. 2A), which indicated that intestinal microenvironment of VS patients may be stimulated by chronic inflammation for a long time. Specifically, as the subcategories, Clostridiales, Lachnospiraceae and three kinds of genera: Intestinibacter, Romboutsia and Roseburia showed the similar significant alterations of microbiome in VS patients, which mostly explained the decreased abundance of Clostridia. Unexpectedly, Eubacterium, which is related to the production of intestinal butyric acid[32] showed higher relative abundance in VS patients compared to EMCS patients. The similar significant were also found at Fusobacteriia, Deltaproteobacteria and their corresponding order and family levels. In addition, Actinomycetaceae, Micrococcaceae, Campylobacteraceae which explained their order levels, performed higher relative abundance in EMCS group than the other two groups (Fig. 2B-C). At genus level, most intestinal microbiota that produce SCFAs or branched-chain fatty acids, like Bacteroides, Blautia, Lactobacillus, Roseburia, Fusobacterium[10,33, 34], showed dramatically low abundance in patients with MCS or VS (Fig. 3A). 
SCFAs regulate the activity of neural cells and benefit for many neurological diseases by gut-microbiota-brain axis[11, 35]. To further explore the SCFAs in the intestinal tract of the three groups, we also detected them in the same fecal sample using targeted lipidomic. In our results, the concentration of acetic acid, propionic acid and butyric acid were significantly decreased in VS group (Fig. 3B-D), which were consistent with the differences in plasma reported in our previous study[19]. In the correlation analysis, Bacteroides, Clostridiales, Blautia were significantly positively correlated with acetic acid or propionic acid. Moreover, some fecal microbiota including valeric acid-associated bacteria (Actinomycetaceae), Roseburia, Bacteroides, were significantly positively correlated with CRS-R scores of the patients, whereas the pathogenic microbial community (Desulfovibrionaceae) showed significantly negatively correlated with it (Fig. 3E). This suggested that there is a causal relationship between the disturbance of microbial community and post-traumatic chronic DoC, which may result from reduction of SCFAs and elevation of pathogenicity determinant.

\section{Predicted function analysis of microbiome in EMCS, MCS, and VS patients}

To further predict functional composition of the intestinal microbiome of chronic DoC patients, PICRUSt was conducted based on KEGG. As shown in Table 2, the broadly potential communication lines were identified between the gut microbiome and patients with EMCS, MCS, VS, including cellular process, metabolism, organism system, human diseases, genetic information processing, environmental information processing. Consistent with the trends in PLS-DA and PCoA models, most level 2 pathways were significantly difference between EMCS group and VS group excluding circulatory system, whereas few pathways like cell growth and death, metabolism of cofactors and vitamins, metabolic diseases, showed differences between EMCS group and MCS group or between MCS group and VS group. Notably, pathways like xenobiotics biodegradation and metabolism, infectious diseases, neurodegenerative diseases enriched in VS group compared to EMCS group, suggesting that patients with low level of consciousness may have high abundance of pathogenic microbiota in their intestines, including some harmful to brain.

\section{Fecal microbiota taxonomic and SCFAs differences between chronic DoC patients treated with and without antibiotics}

Some chronic DoC patients were treated by antibiotics for a long time to prevent infection because of tracheotomy or hypostatic pneumonia[36]. To explore the effect of antibiotics on intestinal microbiome of the patients with chronic DoC, we compared fecal microbiota taxonomic of EMCS group, MCS group, VS group with their corresponding antibiotics treatment groups. The demographic and clinical characteristics of these cohorts are shown in Table S1. To investigate the response of intestinal microbiota to antibiotics in patients with different levels of consciousness, we performed PLS-DA model and found distinct separation among MCS group, VS group and their corresponding antibiotics treatment groups (Fig. 4A). Interestingly, only the MCS group was significantly distinguished from its corresponding antibiotics treatment groups (MCS-Abx) in PCoA model (Fig. 4B) but could not be observed in EMCS and VS groups compared with their corresponding antibiotics treatment groups (EMCS-Abx, VS-Abx) (Supplementary Fig. 2A-B). As a whole, both the mean community richness (ACE and Chao1) and microbial diversity (Shannon and Simpson diversity index) were dramatically decreased in MCS-Abx group compared to MCS group (Fig. 4D), but not in the other groups (Supplementary Fig. 2E-F). Specifically, we compared the taxonomic distributions of fecal microbiota of genus level in MCS and MCS-Abx groups. The 15 genus with the highest relative abundance showed significantly difference between MCS-Abx group and MCS group (Fig. 4C), whereas no significant difference between EMCS and EMCS-Abx, as well as between VS and VS-Abx (Supplementary Fig. 2C-D), suggesting that the intestinal microbiota of MCS patients were more sensitive to antibiotics than those of the EMCS patients and VS patients.

Furthermore, we compared the intestinal microbiota screened by PLS-DA model (VIP>1.4) between MCS group and MCS-Abx group using clustering analysis (Fig 4E). We found most intestinal microbiota decreased and only few ones like Enterococcus and Halomonas enriched in MCS-Abx group in heatmap. We also performed MetaStat analysis to explore the most significantly difference between these two groups (Table S2). Of these, the three groups with the greatest differences: Enterococcus showed higher relative abundance in MCS-Abx group while Blautia and Dialistere did the opposite (Fig 4F). Accordingly, most SCFAs of fecal samples including aceic acid, propionic acid, isobutyric acid, butyric acid, isovaleric acid, dramatically decreased in MCS-Abx group (Fig 4G) and were not significantly different in the other pairwise comparisons (Table S3).

\section{Behavioral scale and EEG reactivity between MCS patients treated with and without antibiotic}

Then, what are the differences of brain function and prognosis between MCS patients treated with and without antibiotics? In our study, the CRS-R scores of all patients showed no significant difference between chronic DoC groups and their corresponding antibiotics treatment groups (Table S1). To find more objective evaluation indicators, we investigated resting EEG spectral power and functional connectivity from part of the patients with intact skulls on the day of fecal collection. As expected, there was also no significant difference of CRS-R scores between the patients in MCS group and MCS-Abx group who had intact skulls and were selected for EEG recording (Fig. 5A). Interestingly, the MCS group showed a higher power of alpha band in the left hemisphere compared to MCS-Abx group, especially the frontal and parietal 
region (Fig. 5B). Furthermore, we screened out the relevant differential electrodes and found that the alpha power of them were significantly lower in MCS-Abx group (Fig. 5C), suggesting that long-term antibiotic use may weaken the alpha band in the frontal parietal lobe of MCS patients. To better measure the difference between the two groups, we assessed functional connectivity including PLI and WPLI, which were used for evaluating the connectivity between brain regions that share functional properties[37] and decreased in many neurological diseases[38, 39]. Using NBS approach and connected topoplot, we observed that the PLI and WPLI were significantly higher in MCS group than MCS-Abx group in alpha band. The significantly stronger connections were mainly located in the left frontal-occipital site. In addition, these two indicators of MCS group were also prominently higher in beta band and connection of the right frontal to left occipital was the most significant (Fig. 5D-5G). To further investigated the effect of long-term antibiotic use on the prognosis of MCS patients, we compared their prognostic scores followed by six months and found that the GOS-E scores of MCS group were higher than MCS-Abx group (Fig. 5H), suggesting that long-term antibiotic use may cause poor prognosis for these patients.

\section{Identification of gut microbiomes related biomarkers to distinguish the EMCS, MCS and VS patients}

To evaluate the diagnostic efficacy of intestinal microbiome in different levels of consciousness, LEfSe analysis was conducted to determine and distinguish the composition of the intestinal microbiota between the EMCS group and MCS group, as well as MCS group and VS group. The intestinal microbiota of the MCS group was dominated by the genera Faecailbacterium, whereas the microbiome of VS group was dominated by the genera Enterococcus and Methanobrevibacter (Fig 6A). We established a diagnostic panel of these three genera to distinguish MCS patients from VS patients and all of these were included in the model using the LASSO algorithm. Then we assessed their diagnostic performance by ROC analysis with an AUC of 0.985 (cut-off value: $0.548, P<0.0001,95 \%$ Cl: 0.951-1.018) (Fig 6B). To validate our candidate biomarkers, they were tested in the validation cohorts (Table S4) and achieved high accuracy (AUC: 0.879 ; cut-off value: 0.443 , $P<0.0005,95 \% \mathrm{Cl}: 0.772-1.019$ ) (Fig 6C). When compared the EMCS group with MCS group, the intestinal microbiota of the former was dominated by Streptococcus and Fusobacterium at genus level, whereas the microbiome of the latter was dominated by the genera Lactococcus, Peptoclostridium, Phascolarctobacterium, Megasphaera (Fig. 6D). We also combined with Streptococcus and Lactococcus that showed the highest LDA scores to distinguish EMCS group from MCS group. Finally, the AUC equaled to 0.844 (cut-off value: $0.609, P=0.0018$, $95 \% \mathrm{Cl}: 0.696-0.993$ ) in discovery cohort and 0.821 (cut-off value: $0.434, P=0.0002,95 \% \mathrm{Cl}: 0.799-1.011$ ) in validation cohort using the LASSO algorithm and ROC analysis (Fig. 6E-F).

To further investigate whether it can be extended to antibiotic cohort, ROC analysis were performed in antibiotic groups. Finally, the AUC equal to 0.895 (cut-off value: $0.017, P=0.0011,95 \% \mathrm{Cl}$ : 0.758-1.033) between MCS-Abx group and VS-Abx group (Supplementary Fig. 3A) and 0.647 (xut-off value: $0.821, P=0.2235,95 \% \mathrm{Cl}: 0.420-0.875$ ) between EMCS-Abx group and MCS-Abx group.

\section{Discussion}

In our study, we identified specific signatures of the fecal microbiota among the patients with different level of consciousness and found that the altered intestinal microbiota was significantly correlated with the clinical behavioral score (CRS-R scores) of the patients and the concentration of SCFAs in their fecal samples. By comparing the groups treated with and without antibiotics, only MCS-Abx group showed dramatically different fecal microbiota and SCFAs relative to MCS group, which were consistent with EEG reactivity of the patients. Finally, we identified the potential diagnostic biomarkers for distinguishing the patients with different level of consciousness both in the discovery cohorts and validation cohorts, which can be also extended to the antibiotic cohort. All these findings indicate that the alteration of intestinal microbiota may play an important role in the identification of patients with different levels of consciousness and influence the recovery of brain function in these patients, especially for MCS patients. To our knowledge, this is the first time to do the intestinal microbiome in chronic DoC patients, as well as explore the effects of antibiotics on their intestinal microbiota and brain function. This study may provide a new perspective for the evaluation and treatment of patients with chronic DoC.

Investigation of intestinal microbiome can explore its relationship to the patients with different level consciousness and provide reference for their nutrition-related intervention. All the patients in our study receive enteral nutrition from the hospital, which minimized the bias for gut microbiomes and lipid metabolism caused by different dietary habits. Different from the TBI patients at acute stage[40], the overall diversity and community richness of intestinal microbiota were not altered among patients in EMCS, MCS and VS, and the same results have shown in those who had experienced mild or moderate/severe TBI several years ago but did not enter into chronic DoC[41, 42]. However, the structure of intestinal microbiota among the three groups showed dramatically different. Some profitable microbial community like Bacteroides, Streptococcus, Roseburia enriched in EMCS patients, whereas some pathogenic microbial community enriched in VS patients, which indicated that the intestinal microecology might not return to its original state, but a new dynamic balance was established when severe DoC patients entered into chronic stage. Currently, only a few probiotics like Lactobacillus or Bifidobacterium are commonly used for some neurological diseases in clinical[43, 44]. More specific probiotics should be developed and applied for chronic DoC patients. Furthermore, acetic acid, propionic acid and butyric acid, which comprise of more than $95 \%$ of the total SCFA pool[10,34] and are produced primarily from dietary fiber, were decreased in VS patients. A large number of studies have shown that SCFAs regulate a growing list of physiological and biochemical 
functions of the host, including gut-brain axis[45] and immunological function[46]. In our previous metabolomics study, we found most of metabolites containing SCFAs were also decreased in plasma of VS patients[19]. Therefore, it is essential to supplement sufficient SCFAs and dietary fiber for these patients. In addition, some differential microbiota showed a high correlation with SCFAs, as well as the CRS-R scores of the patients, which suggested that disturbance of intestinal microbiota and reduction of corresponding SCFAs may influence the recovery of consciousness after brain injury.

Previous study have demonstrated that broad-spectrum antibiotics may decimate the intestinal microbiome[47]. In our study, the patients in antibiotic cohorts were administration of piperacillin, cefoperazone for a long time. Interestingly, only the intestinal microbiome of MCS patients were significantly altered relative to those without antibiotic treated. It might result from the specific baseline microbiota composition of EMCS, MCS and VS groups, which can modulate antibiotic-mediated effects on the gut microbiota and host[48]. Although some pathogenic microbial community like Desulfovibrio and Macrococcus were significantly inhibited, a large number of beneficial bacteria accompanied by its production of SCFAs decreased sharply, which were not conducive to the recovery of the patient's brain function. Furthermore, alpha band is considered of an active role in network coordination and communication[49] and an increase of power and coherence of the frontal and parietal alpha frequency band were observed when the brain of MCS patient was activated[50]. In our study, the power of alpha band of antibiotic cohort was decreased at frontal and parietal region. These brain regions are the critical areas of the default mode network (DMN), which has been shown to reflect the level of consciousness of DoC patients[51]. So, it is possible that the DMN is disrupted indirectly by antibiotics and thereby affecting the recovery of patient's consciousness. On the other hand, the brain network and prognosis were damaged in antibiotic cohort reflected in the weaken of the functional connectivity (PLI and WPLI) and GOS-E scores, which suggested that long-term antibiotic use may affect other consciousness-related networks and the prognosis, even though this performance could not be observed in the CRS-R scale in time. Moreover, the alteration of brain functional connectivity and the poor outcome of MCS patients might explain the loss of abundant profitable microbial community caused by antibiotics. However, further work is needed to identify mechanisms underlying these alterations. In summary, when chronic DoC patients were administered with antibiotics for a long time to prevent infection, their intestinal microecology should also be protected.

Another important highlight of our study is that we identified biomarkers from intestinal microbiome to distinguish between VS and MCS patients, as well as MCS and EMCS patients. Combined with Faecailbacterium, Enterococcus and Methanobrevibacter, we can distinguish the MCS patients from VS patients with high accuracy both in the discovery cohorts and validation cohorts and the diagnosis panel can be extended to the antibiotic cohorts as well. In clinical practice, CRS-R scale is commonly used for diagnosis of VS or MCS patients but lacks sufficient accuracy[52,53]. Although electrophysiology and imaging also can improve the accuracy[54-56], it is difficult to generalize to many patients because of their metal implants and incomplete skulls. Our diagnostic indicators have some advantages, such as non-invasive, convenient. We also investigated the potential biomarkers to distinguish EMCS patients from MCS and found two indicators with sufficient accuracy. However, such conclusions should be replicated in independent study with a large sample size in future.

\section{Conclusion}

In summary, we found that patients with different levels of consciousness have different intestinal microbiome and SCFAs, which can influence brain function especially in MCS patients. In addition, Faecailbacterium, Enterococcus and Methanobrevibacter were considered as potential biomarkers to distinguish patients in MCS from VS with high accuracy both in discovery cohorts and validation cohorts.

\section{Abbreviations}

EMCS: Emerged from Minimally Conscious State; MCS: minimally conscious state; VS: vegetative state; GCS: Glasgow Coma Scale; CRS-R: Coma Recovery Scale-Revised scores; GOS-E: Glasgow Outcome Scale - Extended; GPT: glutamic-pyruvic transaminase; GOT: glutamicpyruvic transaminase; LDH: lactate dehydrogenase; TBI: traumatic brain injury; Abx: antibiotic.

\section{Declarations}

\section{Ethics approval and consent to participate}

This study was approved by the Ethics Committee of the First Affiliated Hospital, School of Medicine, Zhejiang University, Hangzhou Hospital of Zhejiang Armed Police Corps, China, and Hangzhou Mingzhou Brain Rehabilitation Hospital.

\section{Consent for publication}

All authors have approved of the manuscript and agree with its submission.

Availability of data and materials

Page $8 / 20$ 
All intestinal microbiome data are included in the Source Data. The other data sets generated and/or analyzed in the current study are available from the corresponding authors upon reasonable request.

\section{Competing interests}

The authors declare no conflict of interest.

\section{Funding}

This work was supported by grants from the Natural Science Foundation of China (No. 82071173; No. 81901068). This work was supported by Key Realm R\&D Program of Guangzhou (202007030005).

\section{Author Contributions}

B.Y.L and J.Y. conceived the project. J.Y., M.D.L. and B.Y.L. designed the experiments. J.G. and J.Q.L. screened the patients. J.Y., Y.M.Y., X.R.W. and Q.S.C. collected and repackaged stool samples. F.P.H. extracted genome DNA. J. Y. and F.X.M completed microbiome analysis. J.Y., C.X. and G.P. completed EEG analysis. J.Y., M.D.L. and B.Y.L. wrote the manuscript. All authors discussed, reviewed and edited the manuscript. All authors read and approved the final manuscript.

\section{Acknowledgements}

The authors thank the patients and volunteers for participating in this study.

\section{Authors' information}

${ }^{1}$ Department of Neurology, First Affiliated Hospital, School of Medicine, Zhejiang University, Hangzhou 310003, China; ${ }^{2}$ Department of Rehabilitation, Hangzhou Hospital of Zhejiang Armed Police Corps, Hangzhou 310051, China; ${ }^{3}$ Department of Rehabilitation, Hangzhou Mingzhou Brain Rehabilitation Hospital, Hangzhou 311215, China; ${ }^{4}$ State Key Lab of CAD\&CG, Zhejiang University, Hangzhou 310027, China

${ }^{5}$ State Key Laboratory for Diagnosis and Treatment of Infectious Diseases, The First Affiliated Hospital, Collaborative Innovation Center for Diagnosis and Treatment of Infectious Diseases, Zhejiang University School of Medicine, Hangzhou, China

\section{References}

1. Cruse D, Chennu S, Chatelle C, Bekinschtein TA, Fernandez-Espejo D, Pickard JD, Laureys S, Owen AM: Bedside detection of awareness in the vegetative state: a cohort study. Lancet 2011, 378:2088-2094.

2. Monti MM, Laureys S, Owen AM: The vegetative state. BMJ 2010, 341:c3765.

3. Bernat JL: Chronic disorders of consciousness. Lancet 2006, 367:1181-1192.

4. Jennett B, Plum F: Persistent vegetative state after brain damage. A syndrome in search of a name. Lancet 1972, 1:734-737.

5. Machado C: The minimally conscious state: definition and diagnostic criteria. Neurology 2002, 59:1473; author reply $1473-1474$.

6. Chennu S, Annen J, Wannez S, Thibaut A, Chatelle C, Cassol H, Martens G, Schnakers C, Gosseries O, Menon D, Laureys S: Brain networks predict metabolism, diagnosis and prognosis at the bedside in disorders of consciousness. Brain 2017, 140:2120-2132.

7. Bourdillon P, Hermann B, Sitt JD, Naccache L: Electromagnetic Brain Stimulation in Patients With Disorders of Consciousness. Front Neurosci 2019, 13:223.

8. Cryan JF, O'Riordan KJ, Cowan CSM, Sandhu KV, Bastiaanssen TFS, Boehme M, Codagnone MG, Cussotto S, Fulling C, Golubeva AV, et al: The Microbiota-Gut-Brain Axis. Physiol Rev 2019, 99:1877-2013.

9. Morais LH, Schreiber HLt, Mazmanian SK: The gut microbiota-brain axis in behaviour and brain disorders. Nat Rev Microbio/2020.

10. den Besten G, van Eunen K, Groen AK, Venema K, Reijngoud DJ, Bakker BM: The role of short-chain fatty acids in the interplay between diet, gut microbiota, and host energy metabolism. J Lipid Res 2013, 54:2325-2340.

11. Duscha A, Gisevius B, Hirschberg S, Yissachar N, Stangl GI, Eilers E, Bader V, Haase S, Kaisler J, David C, et al: Propionic Acid Shapes the Multiple Sclerosis Disease Course by an Immunomodulatory Mechanism. Cell 2020, 180:1067-1080 e1016. 
13. Liu P, Wu L, Peng G, Han Y, Tang R, Ge J, Zhang L, Jia L, Yue S, Zhou K, et al: Altered microbiomes distinguish Alzheimer's disease from amnestic mild cognitive impairment and health in a Chinese cohort. Brain Behav Immun 2019, 80:633-643.

14. Liu P, Jia XZ, Chen Y, Yu Y, Zhang K, Lin YJ, Wang BH, Peng GP: Gut microbiota interacts with intrinsic brain activity of patients with amnestic mild cognitive impairment. CNS Neurosci Ther 2020.

15. Robba C, Rebora P, Banzato E, Wiegers EJA, Stocchetti N, Menon DK, Citerio G, Collaborative European NeuroTrauma Effectiveness Research in Traumatic Brain Injury P, Investigators: Incidence, Risk Factors, and Effects on Outcome of Ventilator-Associated Pneumonia in Patients With Traumatic Brain Injury: Analysis of a Large, Multicenter, Prospective, Observational Longitudinal Study. Chest 2020, 158:22922303.

16. Yeatts SD, Martin RH, Meurer W, Silbergleit R, Rockswold G, Barsan WG, Korley FK, Wright D, Gajewski BJ: Sliding Scoring of the Glasgow Outcome Scale - Extended (GOS-E) as Primary Outcome in Traumatic Brain Injury Trials. J Neurotrauma 2020.

17. Giacino JT, Kalmar K, Whyte J: The JFK Coma Recovery Scale-Revised: measurement characteristics and diagnostic utility. Arch Phys Med Rehabil 2004, 85:2020-2029.

18. Ishtikhar M, Siddiqui Z, Husain FM, Khan RA, Hassan I: Comparative refolding of guanidinium hydrochloride denatured bovine serum albumin assisted by cationic and anionic surfactants via artificial chaperone protocol: Biophysical insight. Spectrochim Acta A Mol Biomol Spectrosc 2020, 225:117510.

19. Yu J, Meng F, X., He F, P. , Chen F, Bao W, X. , Yu Y, M. , Zhou J, T. , Gao J, Li J, Q. , Yao Y, et al: Metabolic Abnormalities in Patients with Chronic Disorders of Consciousness. Aging and disease 2020, 12.

20. Xiong N, Gao X, Zhao H, Cai F, Zhang FC, Yuan Y, Liu W, He F, Zacharias LG, Lin H, et al: Using arterial-venous analysis to characterize cancer metabolic consumption in patients. Nat Commun 2020, 11:3169.

21. Edgar RC: UPARSE: highly accurate OTU sequences from microbial amplicon reads. Nat Methods 2013, 10:996-998.

22. Caporaso JG, Kuczynski J, Stombaugh J, Bittinger K, Bushman FD, Costello EK, Fierer N, Pena AG, Goodrich JK, Gordon JI, et al: QIIME allows analysis of high-throughput community sequencing data. Nat Methods 2010, 7:335-336.

23. Vogt NM, Kerby RL, Dill-McFarland KA, Harding SJ, Merluzzi AP, Johnson SC, Carlsson CM, Asthana S, Zetterberg H, Blennow K, et al: Gut microbiome alterations in Alzheimer's disease. Sci Rep 2017, 7:13537.

24. Langille MG, Zaneveld J, Caporaso JG, McDonald D, Knights D, Reyes JA, Clemente JC, Burkepile DE, Vega Thurber RL, Knight R, et al: Predictive functional profiling of microbial communities using 16S rRNA marker gene sequences. Nat Biotechno/ 2013, 31:814-821.

25. Poulsen C, Picton TW, Paus T: Agerelated changes in transient and oscillatory brain responses to auditory stimulation in healthy adults 19-45 years old. Cereb Cortex 2007, 17:1454-1467.

26. He F, Wu M, Meng F, Hu Y, Gao J, Chen Z, Bao W, Liu K, Luo B, Pan G: Effects of 20 Hz Repetitive Transcranial Magnetic Stimulation on Disorders of Consciousness: A Resting-State Electroencephalography Study. Neural Plast 2018, 2018:5036184.

27. Nentwich M, Ai L, Madsen J, Telesford QK, Haufe S, Milham MP, Parra LC: Functional connectivity of EEG is subject-specific, associated with phenotype, and different from fMRI. Neuroimage 2020, 218:117001.

28. Stam CJ, Nolte G, Daffertshofer A: Phase lag index: assessment of functional connectivity from multi channel EEG and MEG with diminished bias from common sources. Hum Brain Mapp 2007, 28:1178-1193.

29. Anastasiadou MN, Christodoulakis M, Papathanasiou ES, Papacostas SS, Hadjipapas A, Mitsis GD: Graph Theoretical Characteristics of EEG-Based Functional Brain Networks in Patients With Epilepsy: The Effect of Reference Choice and Volume Conduction. Front Neurosci 2019, 13:221.

30. Zalesky A, Fornito A, Bullmore ET: Network-based statistic: identifying differences in brain networks. Neuroimage 2010, 53:1197-

1207. 
31. Nagata N, Xu L, Kohno S, Ushida Y, Aoki Y, Umeda R, Fuke N, Zhuge F, Ni Y, Nagashimada M, et al: Glucoraphanin Ameliorates Obesity and Insulin Resistance Through Adipose Tissue Browning and Reduction of Metabolic Endotoxemia in Mice. Diabetes 2017, 66:1222-1236.

32. Zaiss MM, Jones RM, Schett G, Pacifici R: The gut-bone axis: how bacterial metabolites bridge the distance. J Clin Invest 2019, 129:3018-3028.

33. Macfarlane S, Macfarlane GT: Regulation of short-chain fatty acid production. Proc Nutr Soc 2003, 62:67-72.

34. Cummings JH, Pomare EW, Branch WJ, Naylor CP, Macfarlane GT: Short chain fatty acids in human large intestine, portal, hepatic and venous blood. Gut 1987, 28:1221-1227.

35. Li JM, Yu R, Zhang LP, Wen SY, Wang SJ, Zhang XY, Xu Q, Kong LD: Dietary fructose-induced gut dysbiosis promotes mouse hippocampal neuroinflammation: a benefit of short-chain fatty acids. Microbiome 2019, 7:98.

36. Robba C, Rebora P, Banzato E, Wiegers EJA, Stocchetti N, Menon DK, Citerio G, Collaborative European NeuroTrauma Effectiveness Research in Traumatic Brain Injury P, Investigators: Incidence, Risk Factors, and Effects on Outcome of Ventilator-Associated Pneumonia in Patients With Traumatic Brain Injury: Analysis of a Large, Multicenter, Prospective, Observational Longitudinal Study. Chest 2020.

37. Biswal BB, Van Kylen J, Hyde JS: Simultaneous assessment of flow and BOLD signals in resting-state functional connectivity maps. NMR Biomed 1997, 10:165-170.

38. Kiiski H, Rueda-Delgado LM, Bennett M, Knight R, Rai L, Roddy D, Grogan K, Bramham J, Kelly C, Whelan R: Functional EEG connectivity is a neuromarker for adult attention deficit hyperactivity disorder symptoms. Clin Neurophysio/ 2020, 131:330-342.

39. Briels CT, Schoonhoven DN, Stam CJ, de Waal H, Scheltens P, Gouw AA: Reproducibility of EEG functional connectivity in Alzheimer's disease. Alzheimers Res Ther 2020, 12:68.

40. Treangen TJ, Wagner J, Burns MP, Villapol S: Traumatic Brain Injury in Mice Induces Acute Bacterial Dysbiosis Within the Fecal Microbiome. Front Immunol 2018, 9:2757.

41. Brenner LA, Stamper CE, Hoisington AJ, Stearns-Yoder KA, Stanislawksi MA, Brostow DP, Hoffmire CA, Forster JE, Schneider AL, Postolache TT, Lowry CA: Microbial Diversity and Community Structures Among Those With Moderate to Severe TBI: A United States-Veteran Microbiome Project Study. J Head Trauma Rehabil 2020, 35:332-341.

42. Urban RJ, Pyles RB, Stewart CJ, Ajami N, Randolph KM, Durham WJ, Danesi CP, Dillon EL, Summons JR, Singh CK, et al: Altered Fecal Microbiome Years after Traumatic Brain Injury. J Neurotrauma 2020, 37:1037-1051.

43. Mazzini L, Mogna L, De Marchi F, Amoruso A, Pane M, Aloisio I, Cionci NB, Gaggia F, Lucenti A, Bersano E, et al: Potential Role of Gut Microbiota in ALS Pathogenesis and Possible Novel Therapeutic Strategies. J Clin Gastroentero/ 2018, 52 Suppl 1, Proceedings from the 9th Probiotics, Prebiotics and New Foods, Nutraceuticals and Botanicals for Nutrition \& Human and Microbiota Health Meeting, held in Rome, Italy from September 10 to 12, 2017:S68-S70.

44. Akkasheh G, Kashani-Poor Z, Tajabadi-Ebrahimi M, Jafari P, Akbari H, Taghizadeh M, Memarzadeh MR, Asemi Z, Esmaillzadeh A: Clinical and metabolic response to probiotic administration in patients with major depressive disorder: A randomized, double-blind, placebocontrolled trial. Nutrition 2016, 32:315-320.

45. Dalile B, Van Oudenhove L, Vervliet B, Verbeke K: The role of short-chain fatty acids in microbiota-gut-brain communication. Nat Rev Gastroenterol Hepatol 2019, 16:461-478.

46. Bachem A, Makhlouf C, Binger KJ, de Souza DP, Tull D, Hochheiser K, Whitney PG, Fernandez-Ruiz D, Dahling S, Kastenmuller W, et al: Microbiota-Derived Short-Chain Fatty Acids Promote the Memory Potential of Antigen-Activated CD8(+) T Cells. Immunity 2019, 51:285-297 e285.

47. Ferrer M, Mendez-Garcia C, Rojo D, Barbas C, Moya A: Antibiotic use and microbiome function. Biochem Pharmacol 2017, 134:114126.

48. Lavelle A, Hoffmann TW, Pham HP, Langella P, Guedon E, Sokol H: Baseline microbiota composition modulates antibiotic-mediated effects on the gut microbiota and host. Microbiome 2019, 7:111.

49. Palva S, Palva JM: New vistas for alpha-frequency band oscillations. Trends Neurosci 2007, 30:150-158.

Page $11 / 20$ 
50. Cavinato M, Genna C, Formaggio E, Gregorio C, Storti SF, Manganotti P, Casanova E, Piperno R, Piccione F: Behavioural and electrophysiological effects of tDCS to prefrontal cortex in patients with disorders of consciousness. Clin Neurophysio/ 2019, 130:231-238.

51. Vanhaudenhuyse A, Noirhomme Q, Tshibanda LJ, Bruno MA, Boveroux P, Schnakers C, Soddu A, Perlbarg V, Ledoux D, Brichant JF, et al: Default network connectivity reflects the level of consciousness in non-communicative brain-damaged patients. Brain 2010, $133: 161-171$.

52. Childs NL, Mercer WN, Childs HW: Accuracy of diagnosis of persistent vegetative state. Neurology 1993, 43:1465-1467.

53. Andrews K, Murphy L, Munday R, Littlewood C: Misdiagnosis of the vegetative state: retrospective study in a rehabilitation unit. BMJ 1996, 313:13-16.

54. Fernandez-Espejo D, Bekinschtein T, Monti MM, Pickard JD, Junque C, Coleman MR, Owen AM: Diffusion weighted imaging distinguishes the vegetative state from the minimally conscious state. Neuroimage 2011, 54:103-112.

55. Yu Y, Meng F, Zhang L, Liu X, Wu Y, Chen S, Tan X, Li X, Kuang S, Sun Y, Luo B: A multi-domain prognostic model of disorder of consciousness using resting-state fMRI and laboratory parameters. Brain Imaging Behav 2020.

56. Erlbeck H, Real RG, Kotchoubey B, Mattia D, Bargak J, Kubler A: Basic discriminative and semantic processing in patients in the vegetative and minimally conscious state. Int J Psychophysiol 2017, 113:8-16.

\section{Tables}

Table 1 Clinical characteristics of patients. 


\begin{tabular}{|c|c|c|c|c|c|c|c|c|}
\hline & \multicolumn{4}{|c|}{ Treat without antibiotics } & \multicolumn{4}{|c|}{ Treat with antibiotics } \\
\hline & EMCS & MCS & VS & $P$ value & EMCS-Abx & MCS-Abx & VS-Abx & $P$ value \\
\hline Patients(n) & 15 & 15 & 13 & / & 9 & 17 & 21 & l \\
\hline Male (n, \%) & $12(80.0 \%)$ & $10(66.7 \%)$ & $9(69.2 \%)$ & 0.691 & $5(55.6 \%)$ & $12(70.6 \%)$ & $15(71.4 \%)$ & 0.668 \\
\hline Age (years) & $52.7 \pm 10.2$ & $49.0 \pm 9.8$ & $58.3 \pm 9.7$ & 0.058 & $61.0 \pm 11.6$ & $53.4 \pm 6.5$ & $54.9 \pm 11.8$ & 0.058 \\
\hline GCS & $7.1 \pm 1.4$ & $6.7 \pm 1.2$ & $7.0 \pm 1.6$ & 0.707 & $6.1 \pm 1.2$ & $6.9 \pm 1.0$ & $7.1 \pm 1.5$ & 0.114 \\
\hline CRS-R & $22.4 \pm 0.8$ & $11.7 \pm 3.1$ & $6.0 \pm 1.3$ & $<0,001^{*}$ & $22.1 \pm 1.4$ & $11.9 \pm 2.9$ & $5.2 \pm 1.3$ & $<0,001^{\star}$ \\
\hline GOS-E & $3.3 \pm 0.5$ & $2.7 \pm 0.8$ & $2.1 \pm 0.6$ & $<0,001^{\star}$ & $3.0 \pm 0.0$ & $2.1 \pm 0.6$ & $2.1 \pm 0.4$ & $<0,001^{\star}$ \\
\hline Length of hospital stay & $172.5 \pm 124.9$ & $158.7 \pm 92.5$ & $101.0 \pm 55.5$ & 0.155 & $79.7 \pm 49.6$ & $120.3 \pm 112.3$ & $169.5 \pm 142.7$ & 0.248 \\
\hline Leucocyte $\left(10^{9} / \mathrm{L}\right)$ & $5.4 \pm 1.7$ & $6.1 \pm 2.0$ & $6.5 \pm 1.8$ & 0.184 & $6.0 \pm 1.4$ & $6.5 \pm 2.1$ & $6.3 \pm 2.1$ & 0.817 \\
\hline Neutrophil $\left(10^{9} / \mathrm{L}\right)$ & $3.7 \pm 1.5$ & $4.2 \pm 1.8$ & $4.3 \pm 1.4$ & 0.503 & $3.7 \pm 1.2$ & $4.7 \pm 2.0$ & $4.4 \pm 1.8$ & 0.415 \\
\hline Lymphocyte $\left(10^{9} / \mathrm{L}\right)$ & $1.3 \pm 0.4$ & $1.3 \pm 0.4$ & $1.3 \pm 0.4$ & 0.931 & $1.4 \pm 0.5$ & $1.3 \pm 0.5$ & $1.2 \pm 0.5$ & 0.649 \\
\hline Monocyte $\left(10^{9} / \mathrm{L}\right)$ & $0.4 \pm 0.2$ & $0.4 \pm 0.2$ & $0.5 \pm 0.2$ & 0.134 & $0.5 \pm 0.1$ & $0.5 \pm 0.3$ & $0.7 \pm 1.2$ & 0.805 \\
\hline Hemoglobin $(\mathrm{g} / \mathrm{L})$ & $124.3 \pm 14.9$ & $124.9 \pm 16.4$ & $111.8 \pm 10.3$ & $0.045^{\star}$ & $117.0 \pm 10.9$ & $105.5 \pm 12.5$ & $115.5 \pm 13.3$ & $0.030 *$ \\
\hline Platelet $\left(10^{9} / \mathrm{L}\right)$ & $219.8 \pm 45.9$ & $199.1 \pm 71.3$ & $236.4 \pm 58.3$ & 0.263 & $209.2 \pm 80.9$ & $252.6 \pm 89.0$ & $221.5 \pm 70.0$ & 0.335 \\
\hline $\begin{array}{l}\text { C-reactive } \\
\text { protein (mg/L) }\end{array}$ & $3.5 \pm 3.7$ & $3.2 \pm 3.3$ & $5.5 \pm 4.3$ & 0.142 & $4.0 \pm 4.4$ & $6.7 \pm 4.0$ & $4.6 \pm 3.5$ & 0.133 \\
\hline GPT (UI/L) & $40.7 \pm 31.5$ & $39.3 \pm 14.6$ & $37.6 \pm 25.4$ & 0.601 & $24.3 \pm 10.9$ & $24.2 \pm 14.2$ & $27.3 \pm 15.6$ & 0.775 \\
\hline GOT (UI/L) & $21.1 \pm 10.6$ & $21.3 \pm 6.0$ & $22.1 \pm 10.0$ & 0.677 & $18.9 \pm 6.6$ & $22.1 \pm 14.9$ & $21.3 \pm 15.3$ & 0.880 \\
\hline LDH (UI/L) & $172.1 \pm 34.5$ & $201.4 \pm 62.7$ & $165.4 \pm 49.0$ & 0.135 & $159.3 \pm 38.3$ & $168.4 \pm 59.8$ & $153.5 \pm 42.9$ & 0.831 \\
\hline Creatinine $(\mu \mathrm{mol} / \mathrm{L})$ & $50.7 \pm 16.1$ & $47.2 \pm 12.9$ & $36.7 \pm 10.0$ & $0.025^{\star}$ & $43.4 \pm 6.9$ & $44.5 \pm 19.1$ & $38.2 \pm 11.1$ & 0.279 \\
\hline Homocysteine $(\mu \mathrm{mol} / \mathrm{L})$ & $12.0 \pm 2.2$ & $9.9 \pm 3.5$ & $10.9 \pm 1.6$ & 0.101 & $9.6 \pm 2.2$ & $7.8 \pm 2.9$ & $8.7 \pm 3.9$ & 0.370 \\
\hline Cause & TBI & TBI & TBI & I & TBI & TBI & TBI & I \\
\hline
\end{tabular}

Continuous variables are expressed as the mean \pm standard deviation (SD); $P<0.05$ was considered statistically significant; GCS: Glasgow Coma Scale; CRS-R: Coma Recovery Scale-Revised scores; GOS-E: Glasgow Outcome Scale - Extended; GPT: glutamic-pyruvic transaminase; GOT: glutamic-pyruvic transaminase; LDH: lactate dehydrogenase; TBI: traumatic brain injury; VS: vegetative state; MCS: minimally conscious state; EMCS: Emerged Minimal Conscious state; Abx: antibiotic.

Table 2 Predicted KEGG functional pathways differences at level 2 inferred from 16S rRNA gene sequences among EMCS, MCS and VS groups using PICRUSt. 


\begin{tabular}{|c|c|c|c|c|c|c|c|}
\hline \multicolumn{2}{|c|}{ KO functional categories } & \multirow{2}{*}{$\begin{array}{l}\text { EMCS } \\
\text { mean\% } \mathrm{GSD} \% \square\end{array}$} & \multirow{2}{*}{$\begin{array}{l}\text { MCS } \\
\text { mean\% } \mathrm{CSD} \% \square\end{array}$} & \multirow{2}{*}{$\begin{array}{l}\text { VS } \\
\text { mean\% } \square S D \% \square\end{array}$} & \multirow{2}{*}{$\begin{array}{l}\text { EMCS } \\
\text { vs } \\
\text { MCS } \\
P \text { value }\end{array}$} & \multirow{2}{*}{$\begin{array}{l}\text { EMCS } \\
\text { vs VS } \\
P \text { value }\end{array}$} & \multirow{2}{*}{$\begin{array}{l}\text { MCS } \\
\text { vs VS } \\
P \text { value }\end{array}$} \\
\hline Level_1 & Level_2 & & & & & & \\
\hline Cellular Processes & Cell Growth and Death & $0.459 \pm 0.045$ & $0.443 \pm 0.053$ & $0.401 \pm 0.042$ & / & 0.002 & 0.029 \\
\hline Metabolism & $\begin{array}{l}\text { Metabolism of Cofactors } \\
\text { and Vitamins }\end{array}$ & $4.128 \pm 0.153$ & $4.107 \pm 0.249$ & $3.911 \pm 0.184$ & / & 0.003 & 0.024 \\
\hline Metabolism & Enzyme Families & $2.166 \pm 0.027$ & $2.142 \pm 0.042$ & $2.107 \pm 0.059$ & / & 0.004 & / \\
\hline Metabolism & $\begin{array}{l}\text { Xenobiotics } \\
\text { Biodegradation and } \\
\text { Metabolism }\end{array}$ & $1.673 \pm 0.115$ & $1.743 \pm 0.191$ & $1.791 \pm 0.087$ & / & 0.005 & / \\
\hline Metabolism & Nucleotide Metabolism & $3.772 \pm 0.225$ & $3.689 \pm 0.365$ & $3.501 \pm 0.244$ & / & 0.006 & / \\
\hline Metabolism & Amino Acid Metabolism & $9.210 \pm 0.352$ & $7.975 \pm 0.703$ & $8.880 \pm 0.342$ & / & 0.018 & / \\
\hline Metabolism & $\begin{array}{l}\text { Biosynthesis of Other } \\
\text { Secondary Metabolites }\end{array}$ & $0.849 \pm 0.098$ & $0.805 \pm 0.098$ & $0.770 \pm 0.076$ & / & 0.024 & / \\
\hline Organismal Systems & Immune System & $0.075 \pm 0.008$ & $0.071 \pm 0.011$ & $0.065 \pm 0.008$ & / & 0.004 & / \\
\hline Organismal Systems & Endocrine System & $0.236 \pm 0.036$ & $0.214 \pm 0.028$ & $0.200 \pm 0.023$ & / & 0.004 & / \\
\hline Organismal Systems & Environmental Adaptation & $0.139 \pm 0.017$ & $0.136 \pm 0.014$ & $0.125 \pm 0.009$ & / & 0.014 & 0.025 \\
\hline Organismal Systems & Nervous System & $0.091 \pm 0.013$ & $0.086 \pm 0.012$ & $0.080 \pm 0.011$ & / & 0.023 & / \\
\hline Organismal Systems & Circulatory System & $0.000 \pm 0.000$ & $0.000 \pm 0.000$ & $0.000 \pm 0.000$ & 0.023 & / & / \\
\hline Human Diseases & Metabolic Diseases & $0.089 \pm 0.006$ & $0.084 \pm 0.005$ & $0.082 \pm 0.005$ & 0.025 & 0.003 & / \\
\hline Human Diseases & Infectious Diseases & $0.436 \pm 0.061$ & $0.456 \pm 0.069$ & $0.499 \pm 0.073$ & / & 0.021 & / \\
\hline Human Diseases & $\begin{array}{l}\text { Neurodegenerative } \\
\text { Diseases }\end{array}$ & $0.108 \pm 0.019$ & $0.111 \pm 0.020$ & $0.124 \pm 0.021$ & / & 0.042 & / \\
\hline $\begin{array}{l}\text { Genetic Information } \\
\text { Processing }\end{array}$ & Replication and Repair & $8.185 \pm 0.488$ & $7.975 \pm 0.103$ & $7.509 \pm 0.451$ & / & 0.001 & 0.045 \\
\hline $\begin{array}{l}\text { Genetic Information } \\
\text { Processing }\end{array}$ & $\begin{array}{l}\text { Folding, Sorting and } \\
\text { Degradation }\end{array}$ & $2.340 \pm 0.057$ & $2.326 \pm 0.055$ & $2.266 \pm 0.071$ & / & 0.006 & 0.023 \\
\hline $\begin{array}{l}\text { Genetic Information } \\
\text { Processing }\end{array}$ & Translation & $5.056 \pm 0.387$ & $3.043 \pm 0.115$ & $4.587 \pm 0.483$ & I & 0.010 & I \\
\hline $\begin{array}{l}\text { Environmental } \\
\text { Information } \\
\text { Processing }\end{array}$ & Membrane Transport & $14.448 \pm 0.870$ & $14.638 \pm 0.849$ & $15.294 \pm 0.730$ & / & 0.010 & 0.037 \\
\hline $\begin{array}{l}\text { Environmental } \\
\text { Information } \\
\text { Processing }\end{array}$ & Signal Transduction & $1.766 \pm 0.255$ & $1.880 \pm 0.315$ & $2.048 \pm 0.289$ & / & 0.012 & / \\
\hline
\end{tabular}

Data are given as mean\% (SD\%). VS: vegetative state; MCS: minimally conscious state; EMCS: Emerged Minimal Conscious state; KEGG: Kyoto Encyclopedia of Genes and Genomes; PICRUSt: Phylogenetic Investigation of Communities by Reconstruction of Unobserved States; KO: KEGG Ortholog; SD: standard deviation

\section{Figures}




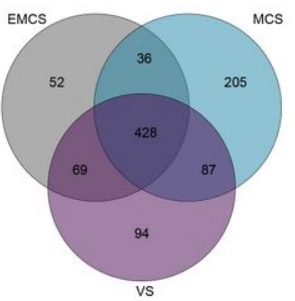

D

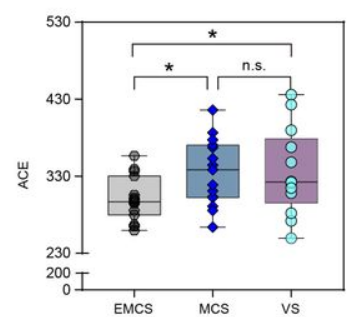

F

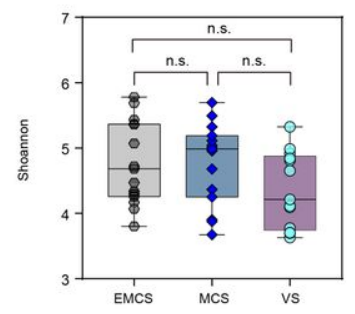

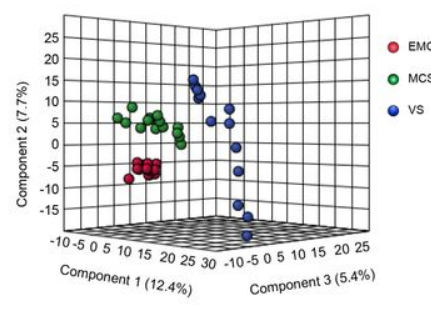

E

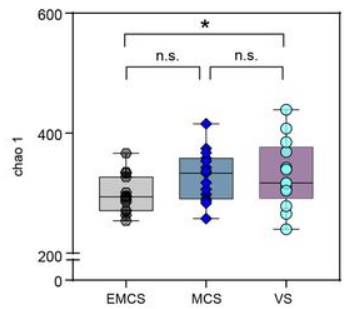

G

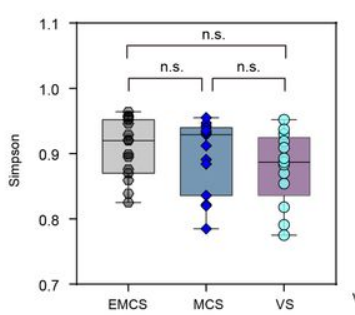

C

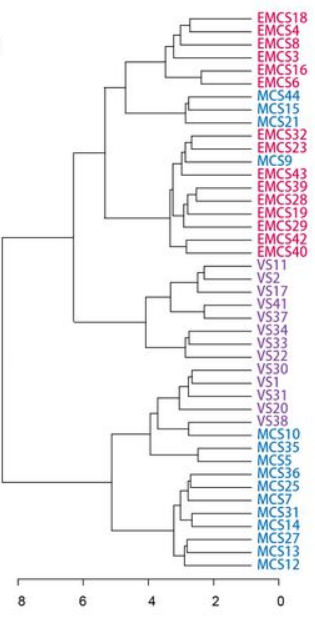

H

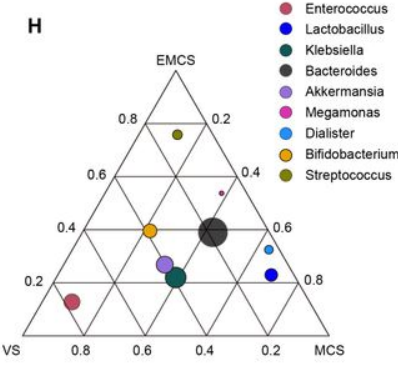

Yu et al., Fig 1

\section{Figure 1}

Changes in fecal microbial diversity and community structures in patients with EMCS, MCS and VS. A) Venn diagram showing the overlap of open taxonomic units of the fecal microbiota across the three groups. B) PLS-DA score plots of the relative abundances of microbiota among EMCS, MCS and VS groups. Three components explain $25.5 \%$ (12.4\%, 5.4\% and 7.7\%) of variance among the three groups. Component scores are indicated as \%; circles indicate individual samples from the EMCS, MCS and VS groups. PLS-DA: partial least-squares discriminant analysis. C) Dendrogram of the three groups (EMCS, MCS, and VS) to show the clustering of all 43 samples. D-G) The a-diversity of the fecal microbiome among three groups depict according to ACE, Chao 1, Shannon index and Simpson index. Each box plot represents the median, interquartile range, minimum, and maximum values. $\mathrm{H}$ ) Ternary plot depicting the number of genera (relative abundance top 10) enriched in the EMCS, MCS, and VS groups. Each circle represents one genus, and the size of the circle reflects relative abundance. P values were determined using a one-way ANOVA or Kruskal-Wallis test, ${ }^{*} \mathrm{P}<0.05$, n.s.: no significant difference. EMCS: Emerged from Minimally Conscious State; MCS: minimally conscious state; VS: vegetative state. 


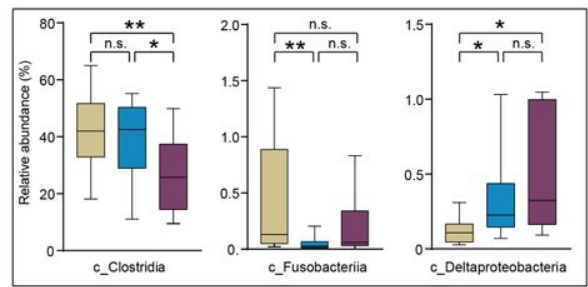

$\square$ EMCS $\square$ MCS

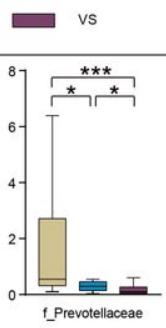

B
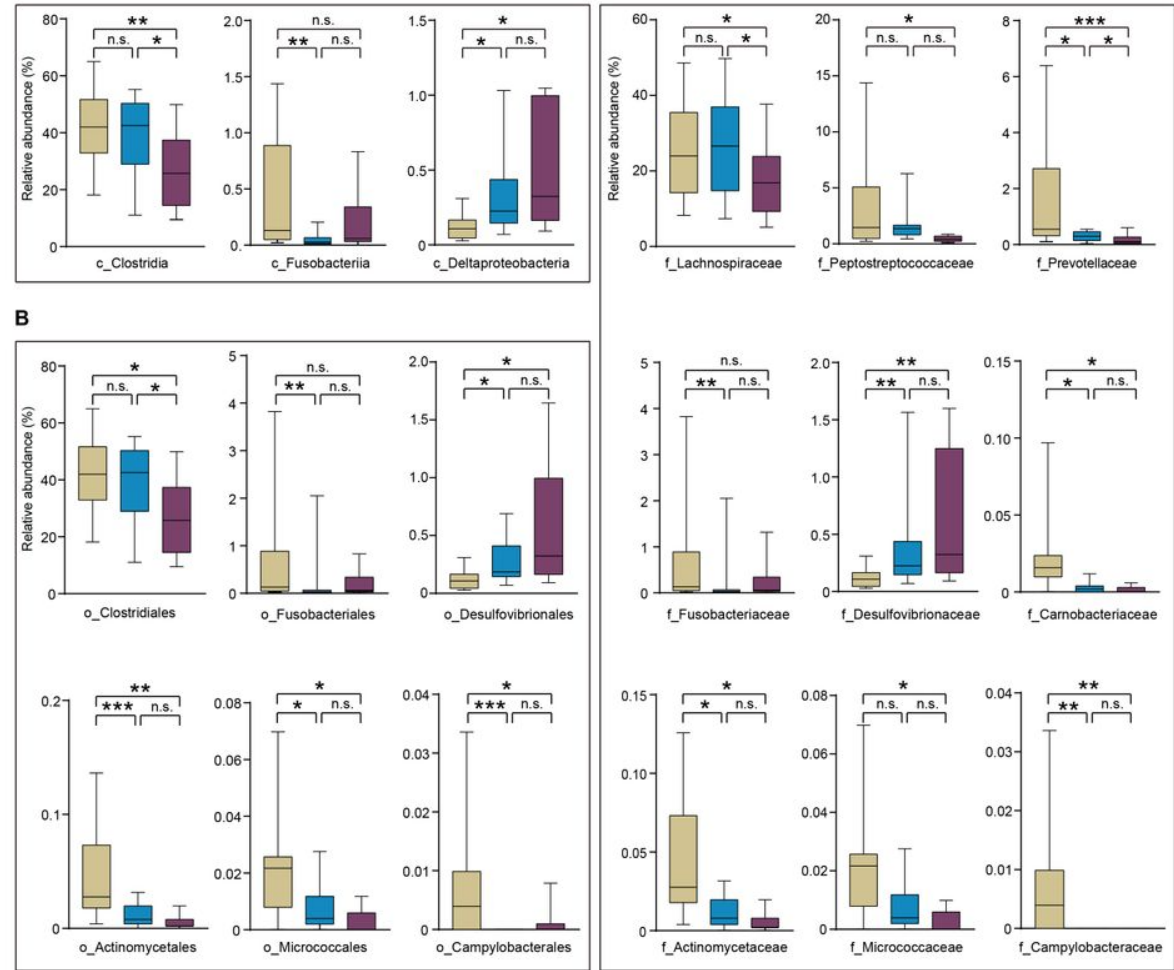

Yu et al., Fig 2

Figure 2

Taxonomic differences of class, family and order level in the fecal microbiota among patients with EMCS, MCS and VS. A) Comparison of the relative abundance at the class level across the three groups. B) Comparison of the relative abundance at the order level across the three groups. C) Comparison of the relative abundance at the family level across the three groups. Each box represents an interquartile range (first and third quartiles) of taxa abundance, and the middle line corresponds to median abundance. No box represents this fecal microbiota cannot be detected in the group. $P$ values are corrected by FDR. FDR: False Discovery Rate. ${ }^{*} P<0.05, * \star P<0.01, * \star * P<0.001$, n.s.: no significant difference. c: class; f: family; o: order; EMCS: Emerged from Minimally Conscious State; MCS: minimally conscious state; VS: vegetative state. 
A

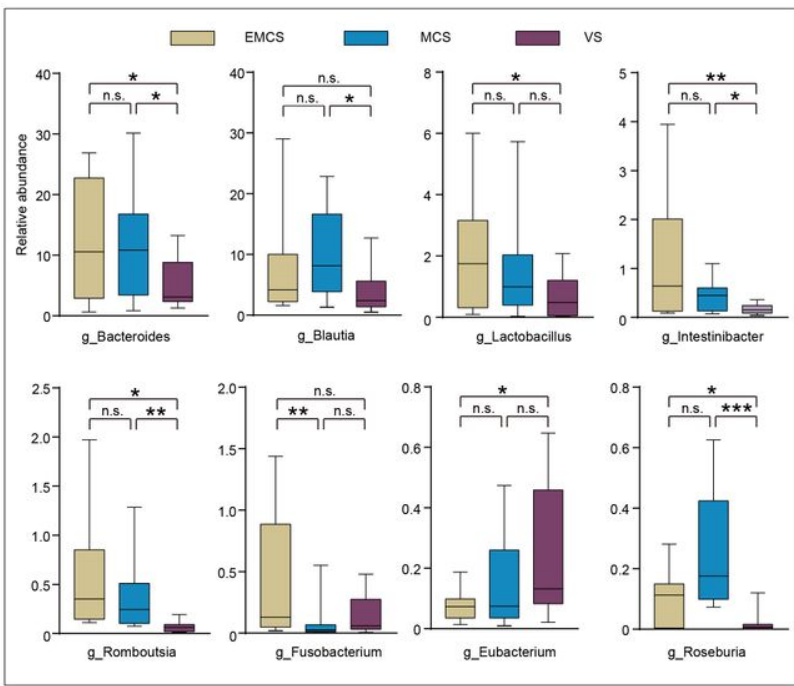

E

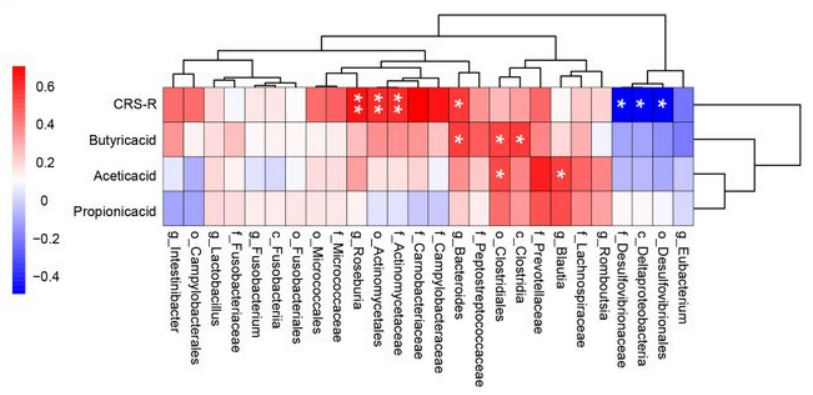

B

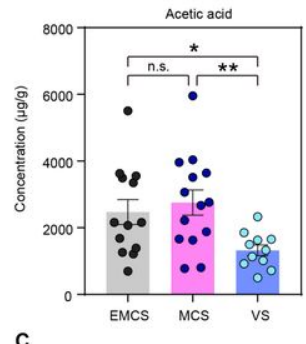

C

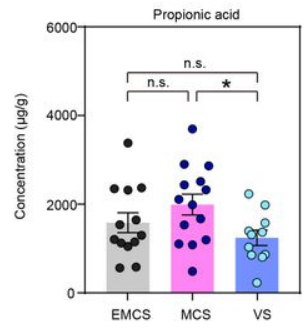

D

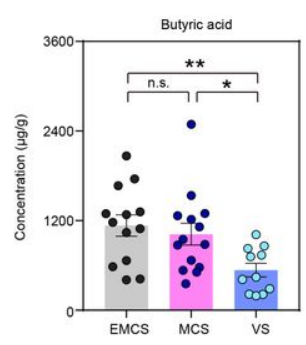

Yu et al., Fig 3

Figure 3

Taxonomic differences of genus level and differences of short-chain fatty acids in the fecal microbiota among patients with EMCS, MCS and VS. A) Comparison of the relative abundance at the genus level across the three groups. Each box represents an interquartile range (first and third quartiles) of taxa abundance, and the middle line corresponds to median abundance. P values are corrected by FDR. FDR: False Discovery Rate. B-D) Comparison of the concentration of differential fecal short-chain fatty acids across the three groups, data represent as the mean \pm SEM. E) Heatmap of Spearman rank correlation analysis between altered fecal microbiota and CRS-R scores, altered short-chain fatty acid, red means positive correlation and blue means negative. P values were determined using a one-way ANOVA or Kruskal-Wallis test, * $P<0.05$, $* * P<0.01$ and ${ }^{* *} P<0.001$, n.s.: no significant difference. g: genera; CRS-R: Coma Recovery Scale-Revised scores; EMCS: Emerged from Minimally Conscious State; MCS: minimally conscious state; VS: vegetative state. 

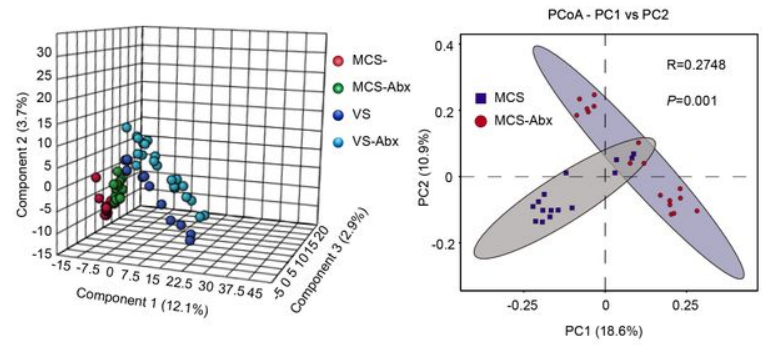

E
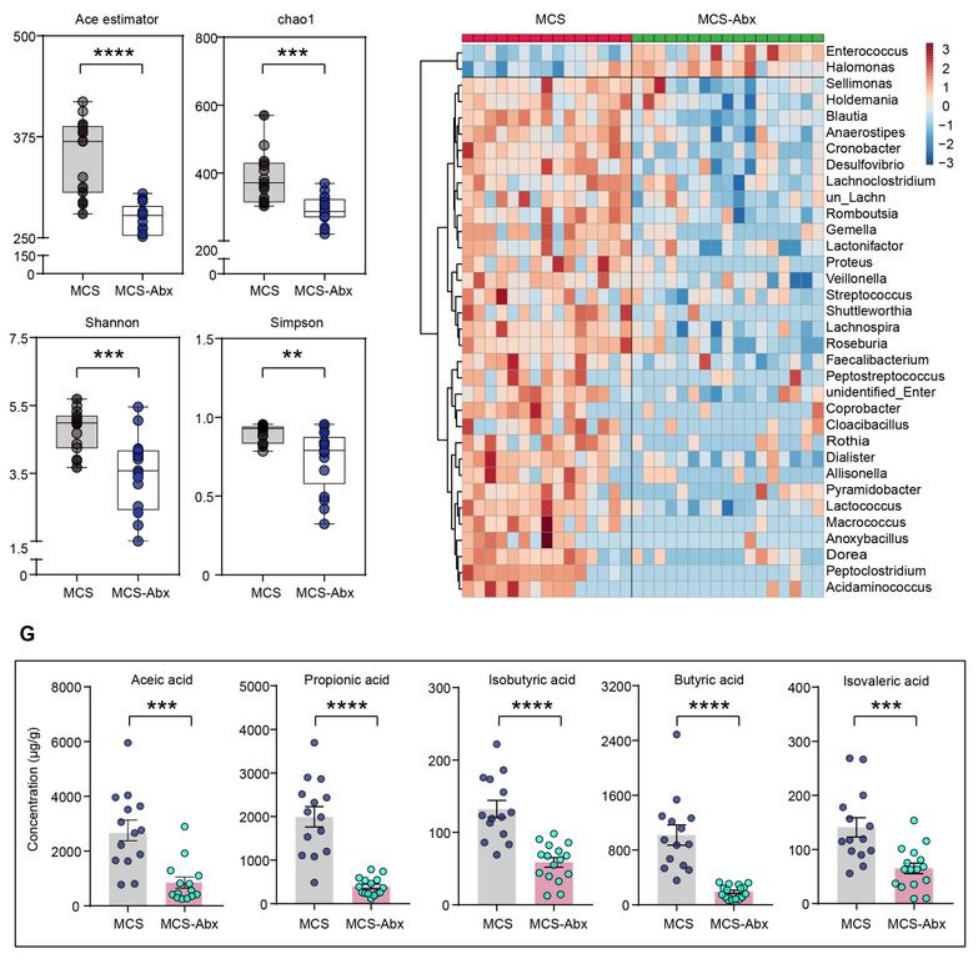

$\mathrm{F}$

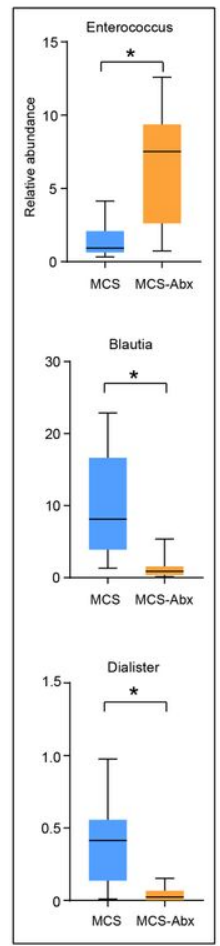

Yu et al., Fig 4

\section{Figure 4}

Comparison of the fecal microbial diversity and community structures in MCS patients treated with or without antibiotics. A) PLS-DA score plots of the relative abundances of microbiota between MCS, MCS-Abx groups and VS, VS-Abx groups. Three components explain $18.7 \%$ $(12.1 \%, 2.9 \%$ and $3.7 \%)$ of variance among the four groups. Component scores are indicated as \%; circles indicate individual samples from the four groups. PLS-DA: partial least-squares discriminant analysis. B) Beta diversity results of the MCS and MCS-Abx groups were assessed by principal coordinate analysis (PCOA); A total of 32 samples and used for PCoA. Two PCs explain $29.5 \%(18.6 \%$ and $10.9 \%)$ of variance between the groups (Bray-Curtis distances: MCS vs. MCS-Abx: $R=0.2748, P=0.001)$. PC scores are indicated as \%; circles indicate individual samples from MCS and MCS-Abx groups. C) Taxonomic distributions of fecal microbiota of genus level in MCS and MCS-Abx groups. D) The a-diversity of the fecal microbiome between MCS and MCS-Abx groups depict according to ACE, Chao 1, Shannon index and Simpson index. Each box plot represents the median, interquartile range, minimum, and maximum values. E) Heatmap of the most abundant metabolites in MCS and MCS-Abx groups, as identified by VIP scores in PLS-DA. Each sample represents a single column. Red color indicates the greater abundance of metabolite. VIP: variable importance in projection. F) Top 3 differential fecal microbiota of genus level by MetaStat analysis. Each box represents an interquartile range (first and third quartiles) of taxa abundance, and the middle line corresponds to median abundance. P values are corrected by FDR, FDR: False Discovery Rate. G) Comparison of the concentration of differential fecal short-chain fatty acids between MCS and MCS-Abx groups, data represent as the mean \pm SEM. P values were determined using Student's t-test or Kruskal-Wallis test, $P$ values are corrected by FDR. FDR: False Discovery Rate, ${ }^{\star} P<0.05,{ }^{\star} * \mathrm{P}<0.01, \star \star \star \mathrm{P}<0.001$, ${ }^{\star \star \star \star} \mathrm{P}<0.0001$ n.s.: no significant difference. MCS: minimally conscious state; VS: vegetative state; Abx: treated with antibiotics. 
A

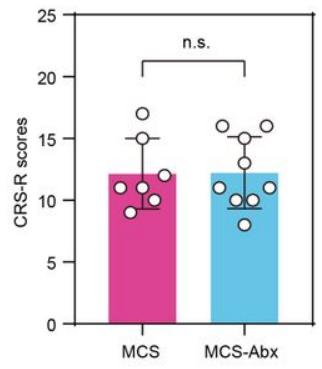

B

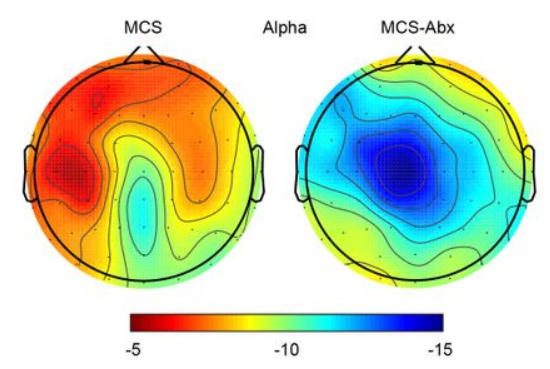

C

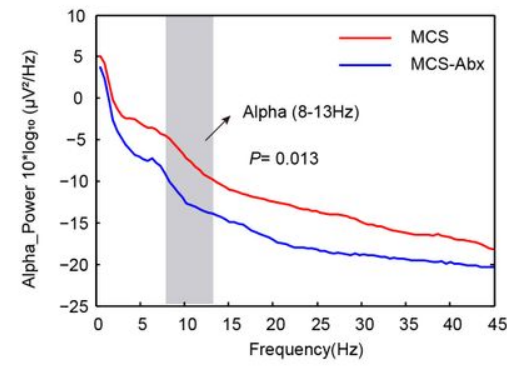

D

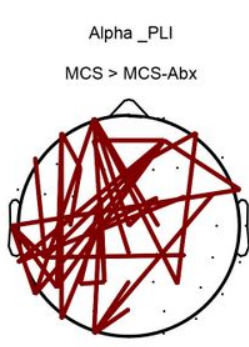

E

Alpha_WPLI

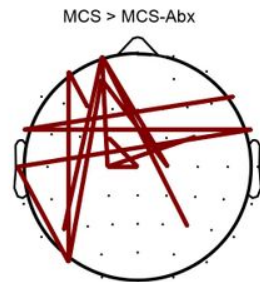

F

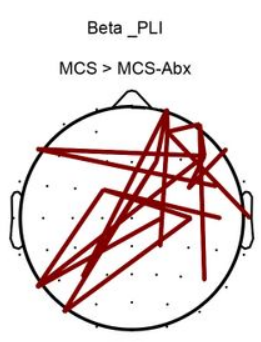

G

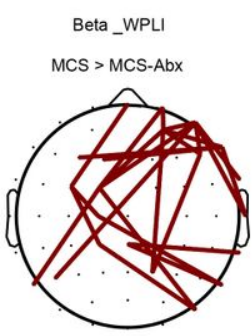

H

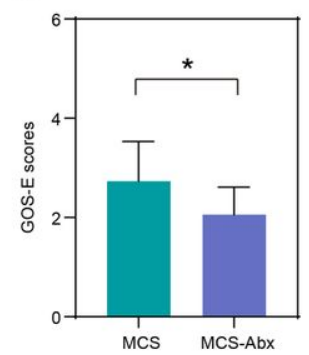

Yu et al., Fig 5

\section{Figure 5}

Comparison of behavioral scale and EEG signals between MCS patients treated with and without antibiotic. A) Comparison of CRS-R scores between the patients conducted EEG recording of MCS and MCS-Abx groups, data represent as the mean \pm SD. B) Topography of the average degree of alpha band power for the two groups. Red color indicates the greater alpha band power. C) Spectral power of MCS and MCS-Abx groups. Averaged alpha power for the differential electrodes (number of sites: 3,5,6,11,17,18,20,21,24,28,30,31,36,38,42,44,48). The vertical grey bar highlights the portion of the graph corresponding to the alpha frequency ranges, both of which showed a significant decreased power in MCS-Abx group ( $P=0.013$ ). Red line represented MCS group and blue line represented MCS-Abx group. D-G) Connected topoplot of PLI and WPLI between MCS and MCS-Abx groups in the alpha and beta bands. NBS to identify interregional connectivity that significantly differed between the groups for the alpha and beta bands. Red lines indicate higher connectivity in MCS group than MCS-Abx group. D: PLI in alpha band ( $P=0.010)$; $E$ : WPLI in alpha band ( $P=0.039)$; F: PLI in beta band $(P=0.002)$; : WPLI in beta band $(P=0.006)$. H) Comparison of $G 0 S-E$ scores followed by six months between MCS and MCS-Abx groups, data represent as the mean \pm SD. P values were determined using Student's t-test or Kruskal-Wallis test, $\mathrm{P}$ values are corrected by FDR. FDR: False Discovery Rate, *P <0.05, n.s.: no significant difference. PLI: phase lag index; WPLI: weighted phase lag index; NBS: network-based statistic; CRS-R: Coma Recovery Scale-Revised scores; GOS-E: Glasgow Outcome Scale - Extended; MCS: minimally conscious state; Abx: treated with antibiotics. 
A

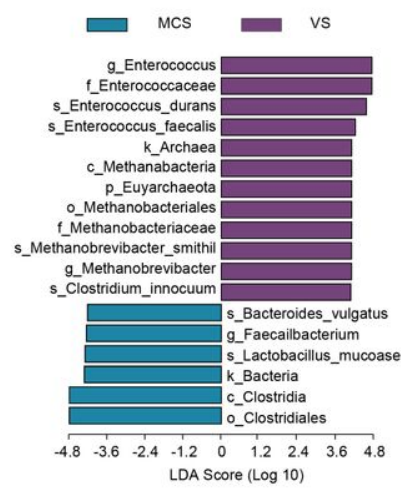

D

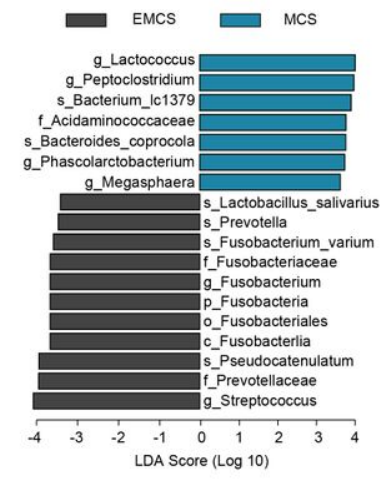

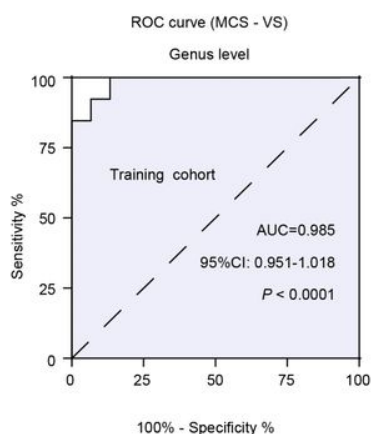

E

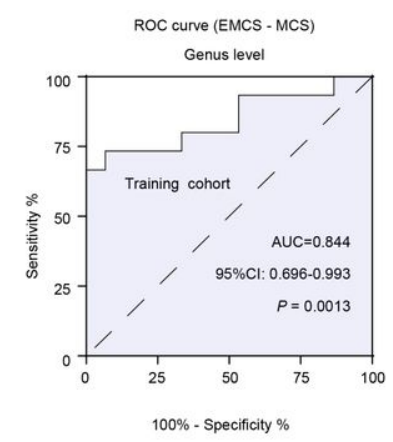

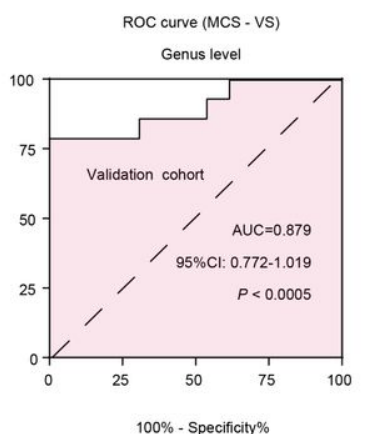

F

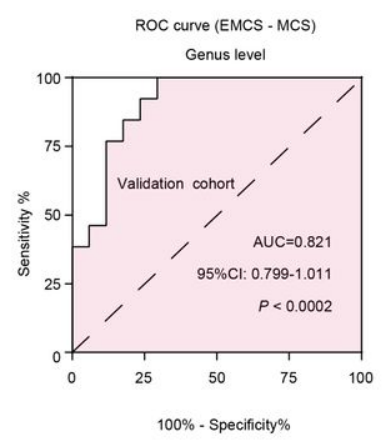

Yu et al., Fig 6

\section{Figure 6}

Linear discrimination analysis (LDA) effect size (LEfSe) analysis of altered fecal microbiota and identification of candidate biomarkers in the discovery cohorts and validation cohorts. A) LDA scores showed significant differences in microbiota composition between MCS group and VS group. Only taxa with an LDA threshold $>4$ are shown. B) ROC curves for Enterococcus, Methanobrevibacter and Faecailbacterium for use in distinguishing MCS patients and VS patients in discovery cohort. C) ROC curves for Enterococcus, Methanobrevibacter and Faecailbacterium for use in distinguishing MCS patients and VS patients in validation cohort. D) LDA scores showed significant differences in microbiota composition between EMCS group and MCS group. Only taxa with an LDA threshold $>3.5$ are shown. E) ROC curves for Lactococcus and Streptococcus for use in distinguishing EMCS patients and MCS patients in discovery cohort. F) ROC curves for Lactococcus and Streptococcus for use in distinguishing EMCS patients and MCS patients in validation cohort. AUC, area under the receiver-operating characteristic curve. EMCS: Emerged from Minimally Conscious State; MCS: minimally conscious state; VS: vegetative state.

\section{Supplementary Files}

This is a list of supplementary files associated with this preprint. Click to download.

- sfig1.jpg

- sfig2.jpg

- sfig3.jpg 